\title{
Neoadjuvant Modified Short-Course Radiotherapy Followed by Delayed Surgery for Locally Advanced Rectal Cancer
}

\author{
Hiroshi Doi 1,2,*(D), Hiroyuki Yokoyama ${ }^{1,3}$, Naohito Beppu ${ }^{4,5}$, Masayuki Fujiwara ${ }^{1,3}$, Shogo Harui ${ }^{1}$, \\ Ayako Kakuno ${ }^{6}$, Hidenori Yanagi ${ }^{4}$, Yoshio Hishikawa ${ }^{1}$, Naoki Yamanaka ${ }^{4}$ and Norihiko Kamikonya ${ }^{1,3}$ \\ 1 Department of Radiation Oncology, Meiwa Cancer Clinic, 3-39 Agenaruocho, Hyogo, \\ Nishinomiya 663-8186, Japan; h-yokoyama@hyo-med.ac.jp (H.Y.); m-fuji@hyo-med.ac.jp (M.F.); \\ harui.shogo@gmail.com (S.H.); hishikawa-yoshio@medipolis.org (Y.H.); \\ kamikonya.n@meiwa-hospital.com (N.K.) \\ 2 Department of Radiation Oncology, Kindai University Faculty of Medicine, 377-2 Ohno-Higashi, \\ Osaka-Sayama, Osaka 589-8511, Japan \\ 3 Department of Radiology, Hyogo College of Medicine, 1-1 Mukogawa-cho, Hyogo, \\ Nishinomiya 663-8501, Japan \\ 4 Department of Surgery, Meiwa Hospital, 4-31 Agenaruo, Hyogo, Nishinomiya 663-8186, Japan; \\ beppu-n@hyo-med.ac.jp (N.B.); yanagih@meiwa-hospital.com (H.Y.); nyamana@meiwa-hospital.com (N.Y.) \\ 5 Division of Lower Gastrointestinal Surgery, Department of Surgery, Hyogo College of Medicine, \\ 1-1 Mukogawa-cho, Hyogo, Nishinomiya 663-8501, Japan \\ 6 Department of Pathology, Meiwa Hospital, 4-31 Agenaruo, Hyogo, Nishinomiya 663-8186, Japan; \\ sugihara@meiwa-hospital.com \\ check for \\ * Correspondence: h-doi@med.kindai.ac.jp; Tel.: +81-72-366-0221; Fax: +81-72-368-2388
}

Citation: Doi, H.; Yokoyama, H.; Beppu, N.; Fujiwara, M.; Harui, S.; Kakuno, A.; Yanagi, H.; Hishikawa, Y.; Yamanaka, N.; Kamikonya, N. Neoadjuvant Modified Short-Course Radiotherapy Followed by Delayed Surgery for Locally Advanced Rectal Cancer. Cancers 2021, 13, 4112. https://doi.org/10.3390/ cancers13164112

Academic Editor: Alessio Giuseppe Morganti

Received: 21 July 2021

Accepted: 13 August 2021

Published: 15 August 2021

Publisher's Note: MDPI stays neutral with regard to jurisdictional claims in published maps and institutional affiliations.

Copyright: (c) 2021 by the authors. Licensee MDPI, Basel, Switzerland. This article is an open access article distributed under the terms and conditions of the Creative Commons Attribution (CC BY) license (https:// creativecommons.org/licenses/by/ $4.0 /)$.
Simple Summary: Both short- and long-course neoadjuvant radiotherapy (NA-RT) followed by surgery have been adopted as standard treatments for locally advanced rectal cancer (LARC). We hypothesized that a modified short-course radiotherapy (mSC-RT) using an accelerated hyperfractionated regimen, with a dose of 2.5 Gy twice daily up to a total dose of 25 Gy in 10 fractions, can provide a favorable therapeutic ratio in comparison with the conventional regimens. Ninety-seven consecutive LARC patients undergoing mSC-RT followed by delayed surgery were analyzed in this retrospective study. Additionally, potential prognostic factors for overall survival (OS) were also assessed. The results showed that mSC-RT followed by delayed surgery achieved equivalent anti-tumor efficacy and acute toxicity that were comparable with long- and short-course NA-RT, respectively. A neutrophil-to-lymphocyte ratio (NLR) $\geq 1.83$ was independently associated with poor OS in LARC patients receiving mSC-RT. Thus, mSC-RT can be a promising alternative to both standard long- and short-course NA-RT regimens.

Abstract: This study aimed to assess the clinical outcomes and predictive factors of neoadjuvant modified short-course radiotherapy (mSC-RT) for locally advanced rectal cancer (LARC). Data from 97 patients undergoing mSC-RT followed by radical surgery for LARC were retrospectively analyzed. A 2.5 Gy dose twice daily up to a total dose of 25 Gy in 10 fractions was administered through mSC-RT, and this was delivered with oral chemotherapy in 95 (97.9\%) patients. Radical surgery was performed 6 (range, 3-13) weeks after mSC-RT. The median follow-up among surviving patients was 43 (8-86) months. All patients completed neoadjuvant radiotherapy with no acute toxicity grade $\geq 3$. Three- and five-year local control rates were $96.3 \%$ and $96.3 \%$, respectively. Three- and five-year overall survival (OS) rates were $92.7 \%$ and $79.8 \%$, respectively. Univariate analyses revealed that poor OS was associated with no concurrent administration of capecitabine, C-reactive-protein-toalbumin ratio $\geq 0.053$, carcinoembryonic antigen $\geq 3.4 \mathrm{ng} / \mathrm{mL}$, and neutrophil-to-lymphocyte ratio $(\mathrm{NLR}) \geq 1.83$ ( $\mathrm{P}=0.045,0.001,0.041$, and 0.001 , respectively). Multivariate analyses indicated that NLR $\geq 1.83$ was independently associated with poor OS $(p=0.018)$. mSC-RT followed by delayed surgery for LARC was deemed feasible and resulted in good clinical outcomes, whereas poor OS was associated with high NLR. 
Keywords: rectal cancer; accelerated hyperfractionated radiotherapy; preoperative radiotherapy; neoadjuvant radiotherapy; short-course radiotherapy; capecitabine; S-1; neutrophil-to-lymphocyte ratio; chemotherapy; radiotherapy

\section{Introduction}

Neoadjuvant radiotherapy (NA-RT) combined with chemotherapy and total mesorectal excision has been adopted as the standard treatment for locally advanced rectal cancer (LARC). Two different approaches of NA-RT are commonly used for LARC: short-course NA-RT (25 Gy in five fractions) and long-course NA-RT (45 to 50.4 Gy in 25 to 28 fractions) [1-4]. The Polish trial and the TROG 01.04 trial have revealed that there are no significant differences in overall survival (OS), local control (LC), distant failure, relapsefree survival (RFS), and late toxicities in randomized controlled trials comparing the shortand long-course NA-RT [5,6]. Moreover, meta-analyses have also shown that there are no significant differences in LC between short-course and long-course NA-RT [7,8].

Short-course NA-RT has several features that are different from those of long-course NA-RT. The efficacy of consolidation chemotherapy following short-course NA-RT is controversial [9]. However, the RAPIDO trial showed that short-course NA-RT had a lower disease-related treatment failure rate than long-course NA-RT when chemotherapy was delivered following NA-RT prior to surgery in both groups [10]. In terms of the interval between short-course NA-RT and surgery, the Stockholm III randomized study compared preoperative short-course RT followed by immediate or delayed surgery and showed a significantly higher pathological complete response rate in the delayed surgery group with an interval of $4-8$ weeks than in the immediate surgery group [11]. They also showed that the postoperative complication rate was significantly lower in the delayed surgery group [12]. Therefore, short-course radiotherapy with delayed surgery has been considered to be a feasible alternative to short-course NA-RT with immediate surgery and long-course NA-RT.

The conventional short-course NA-RT regimen of 25 Gy/5 fractions is reportedly associated with a higher incidence of surgical complications because of the high dose of radiotherapy used per fraction [13]. Delayed surgery could be considered as an option to increase the feasibility of short-course NA-RT. An accelerated hyperfractionated regimen of radiotherapy has been a prospective approach to separate the biologically equivalent dose (BED) between the normal tissue and the tumor and to reduce radiation-induced damages to normal tissues while keeping therapeutic efficacy against cancer cells [14]. Therefore, we hypothesized that short-course NA-RT using an accelerated hyperfractionated regimen for LARC could be highly effective and well-tolerated when compared to conventional short-course NA-RT.

The addition of chemotherapy to long-course NA-RT has been demonstrated to be beneficial, with enhanced tumoricidal effects [15]. The use of 5-fluorouracil (5-FU)-based chemotherapy has gained widespread acceptance for the treatment of LARC [1-4]. Oral fluoropyrimidines, such as capecitabine and S-1, are generally more convenient for patients compared to 5-FU infusions. We have previously reported the tolerability and efficacy of modified short-course radiotherapy (mSC-RT) using an accelerated hyperfractionated regimen that was combined with such oral chemotherapy along with clinical features [16-18].

Prognostic factors in cancer treatment can provide significant information for clinicians and patients to make an appropriate decision when choosing a treatment modality. Several host factors and serum factors have been assessed in rectal cancer [19-22]. The systemic inflammatory response is involved in the progression, treatment response, and prognosis after treatment [20-22]. This systemic inflammatory response can be reflected by hematological parameters such as the neutrophil-to-lymphocyte ratio (NLR) and C-reactiveprotein-to-albumin ratio (CAR), which can be a predictor for oncological outcomes [21,22]. 
However, there are only a few reports showing predictive factors after short-course NA-RT for LARC.

The purpose of this study was to update the clinical outcomes of neoadjuvant mSC-RT for LARC and assess the predictive factors after mSC-RT.

\section{Materials and Methods}

This study was approved by our institutional review board with approval number 2020-26.

\subsection{Patients}

A total of 141 patients with primary rectal adenocarcinoma who underwent NA-RT at Meiwa Cancer Clinic (Hyogo, Japan) between April 2014 and March 2020 were identified in our prospective database. We confirmed the clinical stage at the initial diagnosis for this study based on the 8th edition of the International Union Against Cancer (UICC)/American Joint Committee on Cancer (AJCC) tumor-node-metastasis (TNM) classification system [23]. We excluded the following patients: 8 and 20 patients who had Stage I and IV rectal cancer, respectively; 7 patients who received NA-RT in a conventional fraction; 5 patients who did not undergo surgical resection at our institute; a patient who had a previous irradiation procedure done in the pelvis; and 3 patients who had a follow-up duration of less than 6 months without any specific events. Data from the 97 remaining patients with stage II or III rectal cancer who underwent NA-RT followed by radical surgery at Meiwa Hospital (Hyogo, Japan) were retrospectively analyzed in this study. The patient characteristics are summarized in Table 1.

Table 1. Patient characteristics.

\begin{tabular}{cccc}
\hline & Characteristics & $n=97$ & $(\%)$ \\
\hline Age, years (range) & Male & $66(34-87)$ & \\
Sex & Female & 52 & $(53.6)$ \\
ECOG-PS & 0 & 45 & $(46.4)$ \\
& 1 & 80 & $(82.5)$ \\
& 2 & 15 & $(15.5)$ \\
Localization & 3 & 1 & $(1.0)$ \\
& Ra & 1 & $(1.0)$ \\
Pathological diagnosis & Rb & 23 & $(23.7)$ \\
& & 74 & $(76.3)$ \\
& Tubular adenocarcinoma & 92 & $(94.8)$ \\
& Papillary adenocarcinoma & 3 & $(3.1)$ \\
T & Poorly differentiated adenocarcinoma & 2 & $(2.1)$ \\
& Clinical stage at the diagnosis & & \\
& 1 & 3 & $(3.1)$ \\
N & 2 & 31 & $(32.0)$ \\
& 3 & 50 & $(51.5)$ \\
Stage & 4 & 13 & $(13.4)$ \\
& 0 & 8 & $(8.2)$ \\
& 1 & 53 & $(54.6)$ \\
& 2 & 36 & $(37.1)$ \\
Previous chemotherapy before radiotherapy & III & 9 & $(9.3)$ \\
& Any & 88 & $(90.7)$ \\
& None & 34 & $(35.1)$ \\
& & 63 & $(64.9)$ \\
\hline
\end{tabular}


Table 1. Cont.

\begin{tabular}{lccc}
\hline \multicolumn{2}{c}{ Characteristics } & $n=97$ & $(\%)$ \\
\hline Combined chemotherapy & & & \\
& Capecitabine & 67 & $(69.1)$ \\
& S-1 & 22 & $(22.7)$ \\
& UFT & 5 & $(5.2)$ \\
Adjuvant chemotherapy after surgery & Polysaccharide-K & 1 & $(1.0)$ \\
& none & 2 & $(2.1)$ \\
& Any & 37 & $(38.1)$ \\
\hline
\end{tabular}

${ }^{\dagger}$ Previous chemotherapy included chemotherapy which was performed more than 30 days before radiotherapy. Abbreviations: ECOG-PS, Eastern Cooperative Oncology Group performance status.

\subsection{Treatment}

For radiotherapy, all eligible patients were placed in the supine position and were helically scanned using an Aquilion LB (Canon Medical Systems, Japan) CT unit. For each patient, a planning CT scan of the entire pelvis from the lower-abdomen down until below the ischial tuberosities was obtained at 5-mm intervals. The CT data sets from the FOCUS XiO ${ }^{\mathrm{TM}}$ (Elekta AB, Stockholm, Sweden) between April 2014 and June 2019 and from Monaco version 5.11.03 (Elekta AB, Stockholm, Sweden) from July 2019 onwards were transferred to the treatment planning system to outline the volumes of interest.

The gross target volume (GTV) included the primary rectal tumor and the nodal metastasis, whereas the clinical target volume (CTV) contained the GTV with a $0.5 \mathrm{~cm}$ margin, as well as the perirectal, presacral, and internal iliac nodes. The planning target volume (PTV) was the CTV with a $0.5 \mathrm{~cm}$ margin. Furthermore, there was an additional $7 \mathrm{~mm}$ leaf margin added to the PTV in order to cover the PTV more homogenously. The field margins of each beam were defined and expanded as follows: the cranial margins were the anterior iliac crests or the L4-5 interspace, the caudal margins were the ischial tuberosities, the lateral margins were expanded $1.5 \mathrm{~cm}$ beyond the sacroiliac joint, the anterior margins were the dorsal edge of the pubic joint, and the posterior field margins were designed to include the posterior edge of the sacrum.

Radiotherapy was performed using a three-dimensional conformal technique, which was typically carried out with a 4-field box technique using $10 \mathrm{MV}$ photons. The planned radiotherapy was delivered using an Elekta Synergy (Elekta AB, Stockholm, Sweden) unit.

The patients were treated with a dose of $2.5 \mathrm{~Gy}$ twice daily, with an interval of at least $6 \mathrm{~h}$ between fractions, up to a total dose of $25 \mathrm{~Gy}$ in 10 fractions over one week. The protocol recommended a treatment time of 5 consecutive days from Monday to Friday. S-1 (Taiho Pharmaceutical Co., Tokyo, Japan; $60 \mathrm{mg} / \mathrm{m}^{2} /$ day) between April 2014 and March 2015 or capecitabine (Xeloda; Roche Ltd., Basel, Switzerland; $825 \mathrm{mg} / \mathrm{m}^{2} /$ day) from April 2015 onwards was typically administered orally together with NA-RT. Unless there was lateral lymph nodal metastasis, total mesorectal excision (TME) without prophylactic lateral lymph node dissection was performed 4 weeks after radiotherapy.

\subsection{Analysis}

The data of continuous variables are expressed as medians, with the range in the parentheses, unless otherwise indicated. The time-to-outcome in our study was considered from the start of the radiotherapy to the data of a specific event. Local recurrence was defined as evidence of recurrent disease within the pelvis after a surgical resection. The reported events for RFS were: local recurrence, distant failure, and death. Toxicity was evaluated using the Common Terminology Criteria for Adverse Events (CTCAE) version 5.0 [24].

The pathological tumor response after NA-RT was determined according to the following pathological grading: Grade 0 , not effective; grade $1 \mathrm{a}$, high response in $<1 / 3$ of the cancer cells; grade $1 \mathrm{~b}$, high response in $1 / 3-2 / 3$ of the cancer cells; grade 2 , high response in $>2 / 3$ of the cancer cells; and grade 3 , complete response [25]. In addition, patients with 
grades 2 or 3 tumor response were defined as responders, whereas patients with grades 0 , $1 \mathrm{a}$, or $1 \mathrm{~b}$ tumor response were defined as non-responders.

Time-to-outcome Kaplan-Meier curves were depicted, and the differences in selected populations were analyzed through the log-rank test. The Cox proportional-hazards model was used to evaluate factors that influence OS. The cut-off values of possible serum predictive factors were decided based on the receiver operating characteristic (ROC) curves. The results were reported as hazard ratios with corresponding $95 \%$ confidence intervals (CI). Variables with $p$-values of $<0.20$ according to univariate analysis were analyzed in the multivariate model with Cox regression analysis. The Wilcoxon test was used to compare continuous variables and trends among groups. All statistical analyses were performed using the GraphPad Prism version 8.4.3 (GraphPad Software, Inc., San Diego, CA, USA). The JMP software version 12.2.0 (SAS Institute, Cary, NC, USA) was used to perform Cox regression analysis, and $p$-values of $<0.05$ were considered as statistically significant.

\section{Results}

The median follow-up in all eligible and alive patients was 41 (1-86) and 43 (8-86) months, respectively.

All patients completed NA-RT within 7 days with no cessation. The acute adverse events before surgery are listed in Table 2 . No grade $\geq 3$ toxicity was observed before surgery.

Table 2. Adverse events before surgery.

\begin{tabular}{cccc}
\hline Adverse events per patient & & $n=97$ & \multicolumn{2}{c}{ Incidence (\%) } \\
\hline Any & & 16 & $(16.5)$ \\
\cline { 2 - 4 } Grade & Worst grade per patient & $n=97$ & Incidence (\%) \\
\cline { 2 - 4 } & 2 & 13 & $(13.4)$ \\
None & & 3 & $(3.1)$ \\
\cline { 2 - 4 } & Grade & 81 & $(83.5)$ \\
\hline Adverse events & 1 & $n=97$ & Incidence (\%) \\
Nausea & 2 & 4 & $(4.1)$ \\
Diarrhea & 1 & 1 & $(1.0)$ \\
Enterocolitis & 1 & 5 & $(5.2)$ \\
Fatigue & 2 & 2 & $(1.1)$ \\
Dermatitis & 1 & 1 & $(3.1)$ \\
Anorexia & 2 & 3 & $(1.0)$ \\
Urinary frequency & 1 & 1 & $(1.0)$ \\
Cystitis noninfective & 1 & 1 & $(1.0)$ \\
& 1 & 1 & $(1.0)$ \\
\hline
\end{tabular}

Radical surgery was performed 6 (3-13) weeks after radiotherapy. The sphinctersaving resection was performed in 90 patients $(92.8 \%)$, including one patient who received partial excision surgery. Among 74 patients with lower rectal cancer $(\mathrm{Rb}), 69$ patients (93.2\%) received sphincter-saving surgery. Adjuvant chemotherapy was administered in 37 patients (38.1\%). Except for two patients with pathologically positive surgical margin (R1), complete (R0) resection of the primary tumor was achieved in $95(97.9 \%)$ patients. Additionally, peritoneal dissemination was found intraoperatively in two patients. All four patients who had possible residual cancer cells underwent adjuvant chemotherapy. Pathological responses to neoadjuvant chemoradiotherapy and the stages are shown in Table 3. Pathological complete response was observed in 13 (14.1\%) patients. 
Table 3. Pathological responses to neoadjuvant therapy.

\begin{tabular}{cccc}
\hline Pathological tumor response & $n=92^{*}$ & $(\%)$ \\
\hline Grade & 0 & 1 & $(1.1)$ \\
& $1 \mathrm{a}$ & 21 & $(22.8)$ \\
& $1 \mathrm{~b}$ & 22 & $(38.0)$ \\
& 2 & 35 & $(14.1)$ \\
\hline Pathologic stage & 3 & 13 & \\
\hline T & & & $(13.4)$ \\
& 0 & 13 & $(3.1)$ \\
& Tis & 3 & $(14.4)$ \\
& 1 & 14 & $(27.8)$ \\
& 2 & 27 & $(7.0)$ \\
$\mathrm{N}$ & 3 & 33 & $(63.9)$ \\
& 4 & 7 & $(25.8)$ \\
& 0 & 62 & $(10.3)$ \\
Stage & 1 & 25 & $(15.5)$ \\
& 2 & 10 & $(33.0)$ \\
& 0 & 15 & $(15.5)$ \\
& I & 32 & $(34.0)$ \\
& II & 15 & $(2.1)$ \\
\hline
\end{tabular}

* Excluding five patients in whom pathological grades were not available.

Temporary stoma closing surgery was performed in 80 patients 14 (6-59) weeks after radical surgery, and the stoma was reconstructed in two of them due to pain and fistula formation 13 and 11 months after colostomy closure, respectively. Therefore, 79 of all eligible patients (81.4\%) and 58 patients in the Rb LARC group (78.4\%) could pass material through their anus. Perioperative complications are shown in Table 4. Perioperative complications grade $\geq 3$ were observed in 11 patients $(11.3 \%)$, and one patient experienced aspiration pneumonia resulting in multiple organ failure.

Table 4. Perioperative complications.

\begin{tabular}{cccc}
\hline Adverse events per patient & & $n=97$ & Incidence (\%) \\
\hline Any & & 50 & $(51.5)$ \\
\cline { 2 - 4 } Grade & 1 & $n=97$ & Incidence (\%) \\
\cline { 2 - 4 } & 2 & 5 & $(5.2)$ \\
& 3 & 34 & $(35.1)$ \\
& 4 & 10 & $(10.3)$ \\
Worst grade per patient & 0 & $(0.0)$ \\
None & 5 & 1 & $(1.0)$ \\
\hline Adverse events & & 47 & $(48.5)$ \\
\hline Ileus & Grade & $n=97$ & Incidence (\%) \\
& 1 & 1 & $(1.0)$ \\
& 2 & 11 & $(11.3)$ \\
Dysuria & 3 & 4 & $(1.1)$ \\
& 1 & 1 & $(5.2)$ \\
Fever & 2 & 5 & $(6.2)$ \\
Pelvic infection & 1 & 6 & $(4.1)$ \\
& 2 & 4 & $(1.0)$ \\
Rectal anastomotic leak & 3 & 1 & $(2.1)$ \\
& 2 & 2 & $(2.1)$ \\
\hline
\end{tabular}


Table 4. Cont.

\begin{tabular}{clll}
\hline Colonic fistula & 2 & 1 & $(1.0)$ \\
Colonic obstruction & 3 & 2 & $(2.1)$ \\
Intestinal stoma obstruction & 2 & 3 & $(3.1)$ \\
Dehydration & 2 & 3 & $(3.1)$ \\
Nausea & 2 & 2 & $(2.1)$ \\
Wound infection & 2 & 2 & $(2.1)$ \\
Abdominal infection & 2 & 2 & $(2.1)$ \\
Fatigue & 2 & 2 & $(1.0)$ \\
& 2 & 1 & $(1.0)$ \\
Rectal stenosis & 3 & 1 & $(1.0)$ \\
& 1 & 1 & $(1.0)$ \\
Aspiration & 3 & 1 & $(1.0)$ \\
Prolapse of intestinal stoma & 5 & 1 & $(1.0)$ \\
Postoperative hemorrhage & 3 & 1 & $(1.0)$ \\
Enterocolitis & 3 & 1 & $(1.0)$ \\
Enterocolitis infectious & 2 & 1 & $(1.0)$ \\
Anal pain & 2 & 1 & $(1.0)$ \\
Stoma site infection & 2 & 1 & $(1.0)$ \\
Intestinal stoma leak & 2 & 1 & $(1.0)$ \\
Large intestinal anastomotic leak & 2 & 1 & $(1.0)$ \\
Lymphedema & 2 & 1 & $(1.0)$ \\
Stomal ulcer & 2 & 1 & $(1.0)$ \\
Diarrhea & 2 & 1 & $(1.0)$ \\
Chylous ascites & 2 & 1 & \\
\hline
\end{tabular}

Three patients developed intra-pelvic failure during the follow-up term. The 1-, 3-, and 5-year LC rates were 99.0\%, 96.3\%, and 96.3\%, respectively (Figure 1A). Seventeen patients developed out-of-pelvic failures, including liver metastases, lung metastases, paraaortic nodal metastases, peritoneal dissemination, kidney metastasis, uterine metastasis, and bone metastasis in 11, 8, 3, 1, 1, 1 and 1 patient(s), respectively. Twelve patients died during the follow-up term. Two patients died of small cell lung cancer and pancreatic cancer 45 and 59 months after NA-RT, respectively. The 1-, 3-, and 5-year RFS rates were $85.6 \%, 79.3 \%$, and $72.9 \%$, respectively (Figure 1B). The 1-, 3-, and 5-year OS rates were $96.9 \%, 92.7 \%$, and $79.8 \%$, respectively (Figure $1 \mathrm{C}$ ).

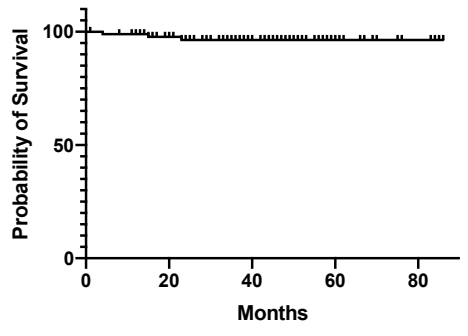

(A)

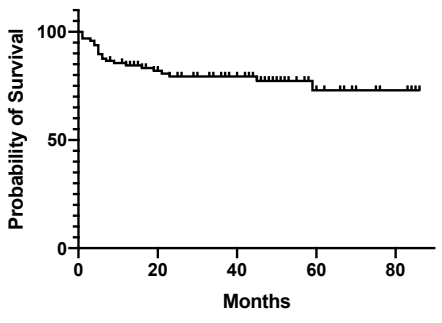

(B)

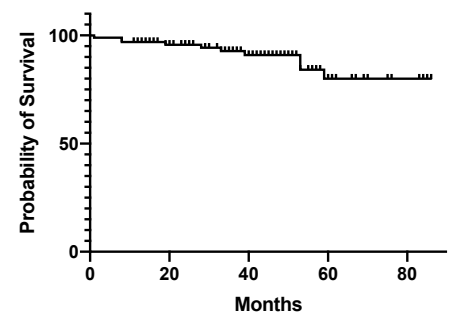

(C)

Figure 1. Clinical outcomes after neoadjuvant radiotherapy. Cumulative rates of local control, relapse-free survival, and overall survival are shown in (A), (B), and (C), respectively. 
The ROC curve indicated cut-off values of $0.053,3.4$, and 1.83 for C-reactive-proteinto-albumin ratio (CAR), carcinoembryonic antigen (CEA), and neutrophil-to-lymphocyte ratio (NLR), respectively (Supplemental Figure S1). Univariate analyses revealed that the following factors were significantly associated with poor OS: no concurrent administration of capecitabine, high CAR, high CEA level, and high NLR (Table 5, Figure 2). Multivariate analysis indicated that high NLR was independently associated with poor OS.

Table 5. Univariate and multivariate analyses for the factors associated with overall survival.

\begin{tabular}{|c|c|c|c|c|c|}
\hline \multirow[b]{2}{*}{ Factors } & \multirow[b]{2}{*}{$n=97$} & \multicolumn{2}{|c|}{ Univariate Analysis } & \multicolumn{2}{|c|}{ Multivariate Analysis } \\
\hline & & Hazard Ratio (95\% CI) & $p$-Value & Hazard Ratio (95\% CI) & $p$-Value \\
\hline \multicolumn{6}{|l|}{ Age (y) } \\
\hline$<65$ & 48 & 1 & 0.191 & 1 & 0.865 \\
\hline$\geq 65$ & 49 & $2.177(0.684-8.168)$ & & $1.137(0.256-5.358)$ & \\
\hline \multicolumn{6}{|l|}{$\overline{\text { Sex }}$} \\
\hline Male & 52 & 1 & 0.309 & & \\
\hline Female & 45 & $1.805(0.576-6.103)$ & & & \\
\hline \multicolumn{6}{|l|}{ ECOG-PS } \\
\hline 0 & 80 & 1 & 0.378 & & \\
\hline $1-$ & 17 & $0.440(0.024-2.264)$ & & & \\
\hline \multicolumn{6}{|l|}{ Location of primary tumor } \\
\hline $\mathrm{Ra}$ & 23 & 1 & 0.456 & & \\
\hline $\mathrm{Rb}$ & 74 & $1.725(0.454-11.226)$ & & & \\
\hline \multicolumn{6}{|l|}{ Primary tumor stage } \\
\hline cT1-3 & 84 & 1 & 0.303 & & \\
\hline cT4 & 13 & $2.083(0.462-7.000)$ & & & \\
\hline \multicolumn{6}{|l|}{ cStage } \\
\hline 2 & 9 & 1 & 0.093 & 1 & 0.306 \\
\hline 3 & 88 & not applicable & & not applicable & \\
\hline \multicolumn{6}{|c|}{ Previous chemotherapy before neoadjuvant radiotherapy } \\
\hline Yes & 34 & 1 & 0.466 & & \\
\hline No & 63 & $0.647(0.206-2.193)$ & & & \\
\hline \multicolumn{6}{|c|}{ Chemotherapy administered with radiotherapy } \\
\hline Capecitabine & $67^{1}$ & 1 & 0.045 & 1 & 0.223 \\
\hline Other & 30 & $3.310(1.027-12.538)$ & & $2.598(0.574-14.103)$ & \\
\hline Capecitabine & 67 & 1 & 0.132 & & \\
\hline S-1 & 23 & $2.667(0.741-10.655)$ & & & \\
\hline \multicolumn{6}{|l|}{ Adjuvant chemotherapy } \\
\hline Yes & 37 & 1 & 0.777 & & \\
\hline No & 60 & $1.189(0.373-4.469)$ & & & \\
\hline \multicolumn{6}{|l|}{$\begin{array}{l}\text { Residual tumor condition after } \\
\text { surgery }\end{array}$} \\
\hline R0 resection & 93 & 1 & 0.084 & 1 & 0.443 \\
\hline $\mathrm{R} 1$ or presence of dissemination & 4 & $4.988(0.765-19.004)$ & & $2.212(0.249-14.846)$ & \\
\hline \multicolumn{6}{|l|}{ Sphincter preservation surgery } \\
\hline Yes & 90 & 1 & 0.230 & & \\
\hline No & 7 & not applicable & & & \\
\hline \multicolumn{6}{|c|}{ Interval between neoadjuvant radiotherapy and surgery } \\
\hline$<6$ weeks & 36 & 1 & 0.165 & 1 & 0.378 \\
\hline$\geq 6$ weeks & 61 & $0.432(0.113-1.404)$ & & $2.082(0.396-10.681)$ & \\
\hline $\begin{array}{l}\text { Pathological response to the } \\
\text { preoperative treatment }\end{array}$ & $n=92$ & & & & \\
\hline Responder & 48 & 1 & 0.806 & & \\
\hline Non-responder & 44 & $1.153(0.360-3.696)$ & & & \\
\hline \multicolumn{6}{|l|}{ Pathological findings } \\
\hline Complete response & 13 & 1 & 0.447 & & \\
\hline \multirow{2}{*}{\multicolumn{6}{|c|}{$\begin{array}{l}\mathrm{C} \text { - reactive-protein-to-albumin } \\
\text { ratio }\end{array}$}} \\
\hline & & & & & \\
\hline$<0.053$ & 59 & 1 & 0.001 & 1 & 0.157 \\
\hline$\geq 0.053$ & 26 & $8.103(2.407-36.632)$ & & $2.801(0.681-14.356)$ & \\
\hline CEA & $n=87$ & & & & \\
\hline$<3.4 \mathrm{ng} / \mathrm{mL}$ & 46 & 1 & 0.041 & 1 & 0.400 \\
\hline$\geq 3.4 \mathrm{ng} / \mathrm{mL}$ & 41 & $3.522(1.050-15.885)$ & & $1.819(0.461-8.924)$ & \\
\hline Neutrophil-to-lymphocyte ratio & $n=90$ & & & & \\
\hline$<1.83$ & 43 & 1 & 0.001 & 1 & 0.018 \\
\hline$\geq 1.83$ & 47 & $12.054(2.328-220.775)$ & & $8.682(1.376-175.625)$ & \\
\hline
\end{tabular}




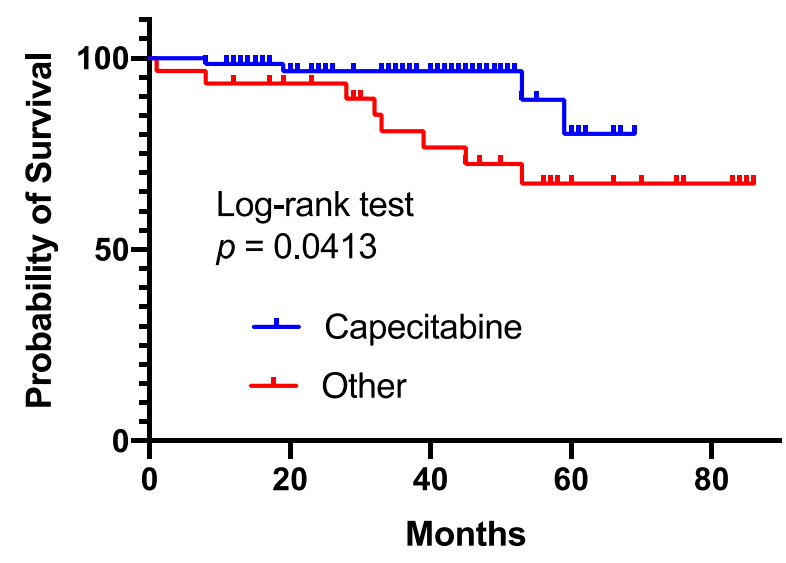

(A)

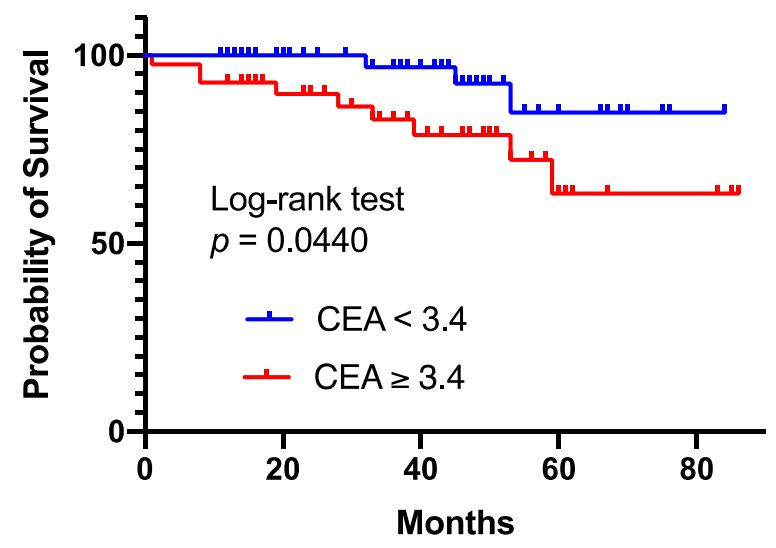

(C)

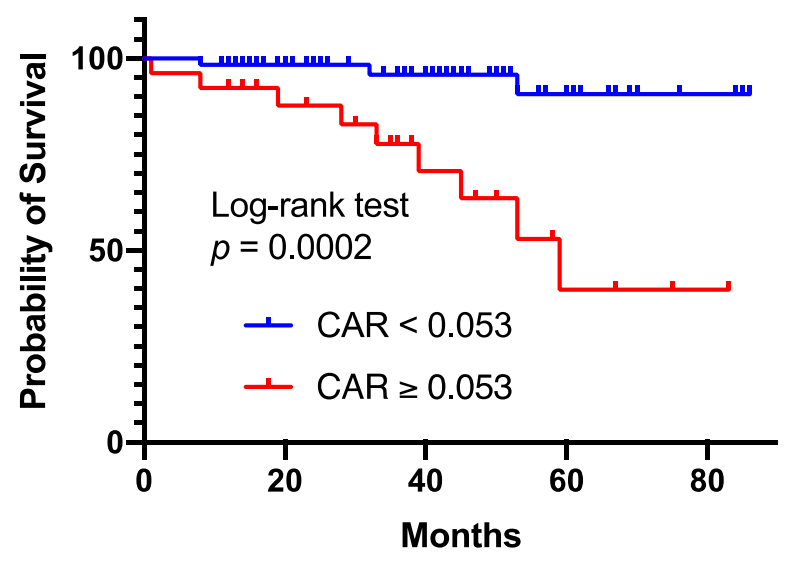

(B)

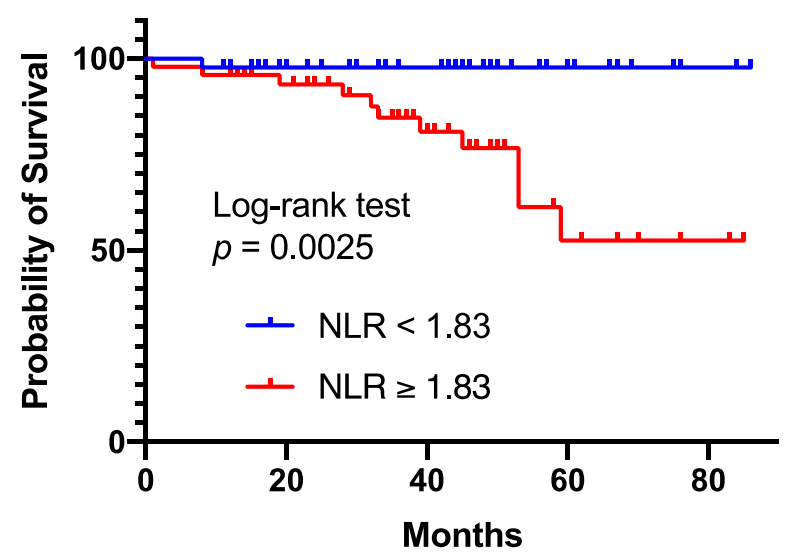

(D)

Figure 2. Overall survivals per each risk factor. Cumulative rates of overall survival for each risk factor, which were identified in univariate analyses using Cox regression analysis. Poor overall survival was significantly associated with no concurrent administration of capecitabine (A), high C-reactive-protein-to-albumin ratio (CAR) (B), high carcinoembryonic antigen (CEA) (C), and high neutrophil-to-lymphocyte ratio (NLR) (D).

Late postoperative adverse events are shown in Table 6. Grade 3 late adverse events were observed in 10 patients $(10.3 \%)$.

Table 6. Postoperative late adverse events.

\begin{tabular}{cccc}
\hline Adverse events per patient & & $n=97$ & \multicolumn{2}{c}{ Incidence (\%) } \\
\hline Any & & 45 & $(46.4)$ \\
\cline { 2 - 4 } & Worst grade per patient & $n=97$ & Incidence (\%) \\
\cline { 2 - 4 } Grade & 1 & 12 & $(12.4)$ \\
& 2 & 23 & $(23.7)$ \\
None & 3 & 10 & $(10.3)$ \\
\cline { 2 - 4 } & & 47 & $(48.5)$ \\
\hline Adverse events & Grade & $n=97$ & Incidence (\%) \\
\hline Diarrhea & 1 & 13 & $(13.4)$ \\
Ileus & 2 & 10 & $(10.3)$ \\
& 1 & 1 & $(1.0)$ \\
& 2 & 3 & $(3.1)$ \\
\hline
\end{tabular}


Table 6. Cont.

\begin{tabular}{clll}
\hline Colonic obstruction & 2 & 2 & $(2.1)$ \\
Wound dehiscence & 3 & 1 & $(1.0)$ \\
Pelvic infection & 2 & 1 & $(1.0)$ \\
Constipation & 3 & 2 & $(1.1)$ \\
& 2 & 1 & $(2.1)$ \\
Gastrointestinal fistula & 3 & 2 & $(2.1)$ \\
Vascular access complication & 1 & 2 & $(1.0)$ \\
Gastrointestinal disorders-other, & 2 & 1 & $(2.1)$ \\
specify (mucosal prolapse) & 3 & 2 & $(1.0)$ \\
Anal pain & 3 & 1 & $(1.0)$ \\
Enterocolitis & 3 & 1 & $(1.0)$ \\
Rectal anastomotic leak & 3 & 1 & $(1.0)$ \\
Erectile dysfunction & 2 & 1 & $(1.0)$ \\
Cystitis noninfective & 2 & 1 & $(1.0)$ \\
Rectal stenosis & 2 & 1 & $(1.0)$ \\
Colonic fistula & 2 & 1 & $(1.0)$ \\
Fatigue & 2 & 1 & $(1.0)$ \\
Anal pain & 1 & 1 & $(1.0)$ \\
\hline
\end{tabular}

\section{Discussion}

In this study, we presented the clinical outcomes of mSC-RT for LARC along with the details of the adverse events and possible predictive factors. Based on the results, mSC-RT was demonstrated to be highly feasible, achieving an excellent complete resection rate and local control rate and a reasonable survival rate. Further, NLR $\geq 1.83$ was revealed as an independent predictor for poor OS in this study.

A multimodal approach has become the standard of care for LARC. In addition, the short-course NA-RT regimen of $25 \mathrm{~Gy} / 5$ fractions is one of the most common regimens [1-13]. The mSC-RT using an accelerated hyperfractionated regimen seems to be a treatment regimen with a favorable therapeutic ratio, with a high BED calculation for tumor in comparison with the conventional short-course NA-RT regimen [14]. The value of $\alpha / \beta$ was $10 \mathrm{~Gy}$ for the rectal tumor. For tumor effects, the BED of the mSC-RT was 31.3 Gy using the formula: $\mathrm{BED}=\mathrm{nd}[1+(\mathrm{d} / \alpha / \beta)]-\gamma / \alpha(\mathrm{T}-\mathrm{Tk})$, where $n$ is the number of fractions and $\mathrm{d}$ is the single fraction dose. Viani et al. have reported in a systematic review that NA-RT with a BED > 30 Gy significantly improved the local control for rectal cancer [26]. Thus, the BED of the present mSC-RT seems to be able to provide the equivalent efficacy for LARC.

The regimen of mSC-RT was combined with oral administration of chemotherapy in most cases in this study. Chemotherapy agents of choice were S-1 from 2014 to 2017 and capecitabine from 2017 onwards due to institutional protocol. The additional benefit of concurrent chemotherapy over short-course NA-RT was proven in only a limited number of prospective randomized trials and is still controversial at present. By itself, mSC-RT may be underpowered in terms of therapeutic efficacy, and thus we hypothesized that oral chemotherapy could enhance the therapeutic benefit of NA-RT for LARC. Response to the conventional NART, followed by surgery weeks later, varied between patients. Complete resection rate, pathological complete response rate, 5-year local control rate, and overall survival rate have been reported to be $90-95 \%, 10-20 \%,>90 \%$, and 60-85\%, respectively $[27,28]$. In addition, the incidence of perioperative complications and a grade 3 late toxicity rate of approximately 10\% were also consistent with those described in previous reports $[5,6,29]$. Furthermore, excellent sphincter preservation rate was also demonstrated in this study [30]. Taken together, mSC-RT followed by delayed surgery achieved equivalent anti-tumor efficacy that was comparable with long-course NA-RT and an acceptable toxicity profile. Notably, acute toxicity was comparable with conventional 
short-course NA-RT, which can be significantly milder than long-course NA-RT. This would be less likely to interfere in the concurrent systemic therapy and can benefit the patients convenience [29]. In addition, NA-RT has recently been recommended in management of elderly patients with LARC [31]. mSC-RT seems considerable in various scenarios because the mild toxicities before surgery were observed in this study. Therefore, we believe mSC-RT can be a promising alternative to both standard long- and short-course NA-RT regimens.

We examined several prognostic factors in this study and observed that poor OS was associated with no concurrent administration of capecitabine, high CAR, high CEA level, and high NLR in univariate analyses. The findings in these serum markers were consistent with previous reports. The use of capecitabine led to a longer survival in comparison with other chemotherapeutic agents or with radiation alone in the Cox hazard model. However, no significant differences were observed between capecitabine and S-1. It has been reported that capecitabine is a recommended agent in combined use with NA-RT [4]. Allegra et al. have demonstrated that the neoadjuvant use of capecitabine, when combined with radiation therapy, is comparable with continuous 5-FU infusion in patients with stage II or III rectal cancer in a phase III randomized trial [32]. Comparing capecitabine and S-1, a phase III randomized trial showed that there were no significant differences in progression-free survival and OS in metastatic colorectal cancers [33]. Sadahiro et al. have reported that the efficacy of S-1 or capecitabine combined with NA-RT for LARC seems to be equivalent [34]. Furthermore, capecitabine was used more recently than S-1 at our institute. This, together with the sophistication of the medical team, supportive care, and systemic chemotherapy, might benefit the OS in patients receiving capecitabine.

We presented that NLR with a cut-off value of 1.83 was a significant predictor for OS in this study. NLR, an inflammatory and immune factor, has been reported as a predictor of OS in various solid tumors, including rectal cancer [21]. However, to the best of our knowledge, there are only a few previous reports indicating the utility of NLR in LARC patients undergoing short-course NA-RT, including mSC-RT combined with chemotherapy. Lymphocytes initially provide protection against cancer cell proliferation and migration. Activated T-cells can be suppressed by marked neutrophil infiltration, and a high NLR could decrease the effects of the lymphocyte-mediated cellular immune response, which could promote cancer progression [20]. Therefore, the present data are consistent with the previous reports that patients with low NLR developed better survival outcomes than those with high NLR. Additionally, a low-range cut-off value (1.83) was obtained in this study. Furthermore, previous reports have indicated that the NLR might predict the effects of immune checkpoint inhibitors in patients with various malignancies [35]. Thus, we hypothesize that patients with high NLR could be candidates for adjuvant chemotherapy and/or target therapy rather than immune checkpoint inhibitors.

We acknowledge that there are several limitations to this study. We presented good outcomes after mSC-RT combined with oral chemotherapy for LARC in a limited number of patients with a relatively short follow-up. However, we were able to present the patients' background, along with the results of the multivariate analysis. In addition, the median follow-up was longer than 3 years, which can be sufficient to evaluate the short-term outcomes.

Some notable types of modern surgical procedures including robotic surgery and transanal TME (TaTME) have been described $[36,37]$. Robotic surgery enables highly flexible and accurate surgery and is expected to improve outcomes over standard laparoscopic surgeries, especially for ultra-low rectal cancer. A meta-analysis has shown that robotic surgery can reduce conversion rates to open surgery, in comparison with laparoscopic surgery [36]. Rectal surgery can be associated with damage to the pelvic organs, leading perioperative complications, and late toxicities, as we described in this study (Tables 4 and 6). TaTME has been described in challenging cases with the aim of radical resection, the preservation of pelvic nerves, and the achievement of a restorative procedure [37]. Preferable surgical procedures following mSC-RT should be investigated and might improve clinical 
outcomes, although we demonstrated favorable therapeutic outcomes along with a high tolerability in this study.

Based on the present study, we believe that mSC-RT combined with oral chemotherapy could be an alternative to the conventional long- and short-course NA-RT for LARC. Longer follow-up and a prospective controlled study should be performed in the future in order to clarify the efficacy of mSC-RT.

\section{Conclusions}

To conclude, we herein presented that mSC-RT for LARC was well tolerated by the patients and produced excellent clinical outcomes. Poor OS was associated with high NLR. As such, mSC-RT can be a promising alternative to conventional long- and short-course NA-RT regimens. Further trials with larger numbers of homogenous patients with LARC and longer follow-up periods are warranted based on our findings.

Supplementary Materials: The following are available online at https://www.mdpi.com/article/ 10.3390/cancers13164112/s1, Figure S1: Receiver operating characteristic (ROC) curves to identify cut-off values for possible serum predictive factors. ROC curves were generated to identify cut-off values for possible serum predictive factors including C-reactive protein to albumin ratio (CAR) (A), carcinoembryonic antigen (CEA) (B), and neutrophil-to-lymphocyte ratio (NLR) (C). Area under the ROC curve was $0.71,0.66$, and 0.68 in $\mathrm{A}, \mathrm{B}, \mathrm{C}$, respectively.

Author Contributions: Conceptualization, H.D.; Methodology, H.D.; Software, H.D.; Validation, H.D., H.Y. (Hiroyuki Yokoyama) and N.B.; Formal Analysis, H.D.; Investigation, H.D., H.Y. (Hiroyuki Yokoyama), N.B., M.F., S.H., A.K., H.Y. (Hidenori Yanagi), Y.H. and N.K.; Resources, H.D.; Data Curation, H.D. and H.Y. (Hiroyuki Yokoyama); Writing-Original Draft Preparation, H.D.; Writing-Review and Editing, N.B. and M.F.; Visualization, H.D.; Supervision, H.Y. (Hidenori Yanagi), Y.H., N.Y. and N.K.; Project Administration, H.D.; Funding Acquisition, H.D. All authors read and approved the final manuscript.

Funding: This work was supported by JSPS KAKENHI Grant Number JP20K08093.

Institutional Review Board Statement: This study was conducted according to the guidelines of the Declaration of Helsinki. This study was approved by our institutional review board with approval number 2020-26.

Informed Consent Statement: Written informed consent for radiotherapy was obtained from all individual participants prior to radiotherapy. Informed consent on this study was obtained in the form of opt-out.

Data Availability Statement: The data are available from the corresponding authors upon reasonable request.

Conflicts of Interest: The authors have no conflict of interest to declare.

\section{References}

1. Oronsky, B.; Reid, T.; Larson, C.; Knox, S.J. Locally Advanced Rectal Cancer: The Past, Present, and Future. Semin. Oncol. 2020, 47, 85-92. [CrossRef]

2. Argilés, G.; Tabernero, J.; Labianca, R.; Hochhauser, D.; Salazar, R.; Iveson, T.; Laurent-Puig, P.; Quirke, P.; Yoshino, T.; Taieb, J.; et al. Localised colon cancer: ESMO Clinical Practice Guidelines for diagnosis, treatment and follow-up. Ann. Oncol. 2020, 31, 1291-1305. [CrossRef] [PubMed]

3. Wo, J.Y.; Anker, C.J.; Ashman, J.B.; Bhadkamkar, N.A.; Bradfield, L.; Chang, D.T.; Dorth, J.; Garcia-Aguilar, J.; Goff, D.; Jacqmin, D.; et al. Radiation Therapy for Rectal Cancer: Executive Summary of an ASTRO Clinical Practice Guideline. Pract. Radiat. Oncol. 2021, 11, 13-25. [CrossRef] [PubMed]

4. NCCN Clinical Practice Guidelines in Oncology (NCCN Guidelines ${ }^{\circledR}$ ) Rectal Cancer Version 1. 2021. Available online: https: //www.nccn.org/professionals/physician_gls/pdf/rectal.pdf (accessed on 6 July 2021).

5. Ngan, S.Y.; Burmeister, B.; Fisher, R.J.; Solomon, M.; Goldstein, D.; Joseph, D.; Ackland, S.P.; Schache, D.; Mcclure, B.; Mclachlan, S.; et al. Randomized Trial of Short-Course Radiotherapy Versus Long-Course Chemoradiation Comparing Rates of Local Recurrence in Patients With T3 Rectal Cancer: Trans-Tasman Radiation Oncology Group Trial 01.04. J. Clin. Oncol. 2012, 30, 3827-3833. [CrossRef] [PubMed] 
6. Bujko, K.; Nowacki, M.P.; Nasierowska-Guttmejer, A.; Michalski, W.; Kryj, M. Long-term results of a randomized trial comparing preoperative short-course radiotherapy with preoperative conventionally fractionated chemoradiation for rectal cancer. $\mathrm{Br}$. $\mathrm{J}$. Surg. 2006, 93, 1215-1223. [CrossRef] [PubMed]

7. Socha, J.; Kairevice, L.; Kepka, L.; Michalski, W.; Spałek, M.; Paciorek, K.; Bujko, K. Should Short-Course Neoadjuvant Radiation Therapy Be Applied for Low-Lying Rectal Cancer? A Systematic Review and Meta-Analysis of the Randomized Trials. Int. J. Radiat. Oncol. Biol. Phys. 2020, 108, 1257-1264. [CrossRef]

8. Chen, C.; Sun, P.; Rong, J.; Weng, H.-W.; Dai, Q.; Ye, S. Short Course Radiation in the Treatment of Localized Rectal Cancer: A Systematic Review and Meta-Analysis. Sci. Rep. 2015, 5, 10953. [CrossRef]

9. Ciseł, B.; Pietrzak, L.; Michalski, W.; Wyrwicz, L.; Rutkowski, A.; Kosakowska, E.; Cencelewicz, A.; Spałek, M.; Polkowski, W.; Jankiewicz, M.; et al. Long-course preoperative chemoradiation versus $5 \times 5$ Gy and consolidation chemotherapy for clinical T4 and fixed clinical T3 rectal cancer: Long-term results of the randomized Polish II study. Ann. Oncol. 2019, 30, 1298-1303. [CrossRef] [PubMed]

10. Bahadoer, R.R.; Dijkstra, E.A.; van Etten, B.; Marijnen, C.A.M.; Putter, H.; Kranenbarg, E.M.-K.; Roodvoets, A.G.H.; Nagtegaal, I.D.; Beets-Tan, R.G.H.; Blomqvist, L.K.; et al. Short-course radiotherapy followed by chemotherapy before total mesorectal excision (TME) versus preoperative chemoradiotherapy, TME, and optional adjuvant chemotherapy in locally advanced rectal cancer (RAPIDO): A randomised, open-label, phase 3 trial. Lancet Oncol. 2021, 22, 29-42. [CrossRef]

11. Pettersson, D.; Lörinc, E.; Holm, T.; Iversen, H.; Cedermark, B.; Glimelius, B.; Martling, A. Tumour regression in the randomized Stockholm III Trial of radiotherapy regimens for rectal cancer. Br. J. Surg. 2015, 102, 972-978. [CrossRef]

12. Erlandsson, J.; Holm, T.; Pettersson, D.; Berglund, Å.; Cedermark, B.; Radu, C.; Johansson, H.; Machado, M.; Hjern, F.; Hallböök, O.; et al. Optimal fractionation of preoperative radiotherapy and timing to surgery for rectal cancer (Stockholm III): A multicentre, randomised, non-blinded, phase 3, non-inferiority trial. Lancet Oncol. 2017, 18, 336-346. [CrossRef]

13. Peeters, K.C.M.J.; van de Velde, C.J.H.; Leer, J.W.H.; Martijn, H.; Junggeburt, J.M.C.; Kranenbarg, E.K.; Steup, W.H.; Wiggers, T.; Rutten, H.J.; Marijnen, C.A.M. Late side effects of short-course preoperative radiotherapy combined with total mesorectal excision for rectal cancer: Increased bowel dysfunction in irradiated patients-a Dutch colorectal cancer group study. J. Clin. Oncol. 2005, 23, 6199-6206. [CrossRef] [PubMed]

14. Withers, H.R. Radiation biology and treatment options in radiation oncology. Cancer Res. 1999, 59, 1676s-1684s. [PubMed]

15. Ceelen, W.; Fierens, K.; Nieuwenhove, Y.V.; Pattyn, P. Preoperative chemoradiation versus radiation alone for stage II and III resectable rectal cancer: A systematic review and meta-analysis. Int. J. Cancer 2009, 124, 2966-2972. [CrossRef]

16. Beppu, N.; Matsubara, N.; Kakuno, A.; Doi, H.; Kamikonya, N.; Yamanaka, N.; Yanagi, H.; Tomita, N. Feasibility of modified short-course radiotherapy combined with a chemoradiosensitizer for T3 rectal cancer. Dis. Colon Rectum 2015, 58, $479-487$. [CrossRef]

17. Beppu, N.; Kimura, F.; Aihara, T.; Doi, H.; Tomita, N.; Yanagi, H.; Yamanaka, N. Patterns of Local Recurrence and Oncologic Outcomes in T3 Low Rectal Cancer ( $\leq 5 \mathrm{~cm}$ from the Anal Verge) Treated With Short-Course Radiotherapy With Delayed Surgery. Ann. Surg. Oncol. 2017, 24, 219-226. [CrossRef] [PubMed]

18. Beppu, N.; Kimura, F.; Okamoto, R.; Nakamoto, Y.; Tomita, N.; Yanagi, H.; Yamanaka, N. Early results of a phase-II study of modified short-course radiotherapy combined with capecitabine and delayed surgery for T3M0 lower rectal cancer. Acta Oncol. 2018, 57, 860-862. [CrossRef] [PubMed]

19. Divitiis, C.D.; Nasti, G.; Montano, M.; Fisichella, R.; Iaffaioli, R.V.; Berretta, M. Prognostic and predictive response factors in colorectal cancer patients: Between hope and reality. World J. Gastroenterol. 2014, 20, 15049-15059. [CrossRef]

20. Diakos, C.I.; Charles, K.A.; McMillan, D.C.; Clarke, S.J. Cancer-Related Inflammation and Treatment Effectiveness. Lancet Oncol. 2014, 15, e493-e503. [CrossRef]

21. Zhang, Y.; Liu, X.; Xu, M.; Chen, K.; Li, S.; Guan, G. Prognostic value of pretreatment systemic inflammatory markers in patients with locally advanced rectal cancer following neoadjuvant chemoradiotherapy. Sci. Rep. 2020, 10, 8017. [CrossRef]

22. Shibutani, M.; Maeda, K.; Nagahara, H.; Iseki, Y.; Ikeya, T.; Hirakawa, K. Prognostic Significance of the Preoperative Ratio of C-Reactive Protein to Albumin in Patients with Colorectal Cancer. Anticancer Res. 2016, 3, 995-1001.

23. Classification of Malignant Tumours, 8th ed.; Brierley, J.D.; Gospodarowicz, M.K.; Christian Wittekind, C. (Eds.) International Union Against Cancer: Geneva, Switzerland; Wiley-Blackwell: Hoboken, NJ, USA, 2016.

24. Common Terminology Criteria for Adverse Events (CTCAE) v5.0. Available online: https: / ctep.cancer.gov/ protocoldevelopment/ electronic_applications/docs/ctcae_v5_quick_reference_5x7.pdf (accessed on 17 April 2021).

25. Japanese Society for Cancer of the Colon and Rectum. Japanese Classification of Colorectal, Appendiceal and Anal Carcinoma: The 3d English Edition [Secondary Publication]. J. Anus Rectum Colon 2019, 3, 175-195. [CrossRef]

26. Viani, G.A.; Stefano, E.J.; Soares, F.V.; Afonso, S.L. Evaluation of Biologic Effective Dose and Schedule of Fractionation for Preoperative Radiotherapy for Rectal Cancer: Meta-Analyses and Meta-Regression. Int. J. Radiat. Oncol. Biol. Phys. 2011, 80, 985-991. [CrossRef] [PubMed]

27. Jin, F.; Luo, H.; Zhou, J.; Wu, Y.; Sun, H.; Liu, H.; Zheng, X.; Wang, Y. Dose-time fractionation schedules of preoperative radiotherapy and timing to surgery for rectal cancer. Ther. Adv. Med. Oncol. 2020, 12, 1758835920907537. [CrossRef]

28. Feeney, G.; Sehgal, R.; Sheehan, M.; Hogan, A.; Regan, M.; Joyce, M.; Kerin, M. Neoadjuvant radiotherapy for rectal cancer management. World J. Gastroenterol. 2019, 25, 4850-4869. [CrossRef] 
29. Ansari, N.; Solomon, M.J.; Fisher, R.J.; Mackay, J.; Burmeister, B.; Ackland, S.; Heriot, A.; Joseph, D.; Mclachlan, S.; Mcclure, B.; et al. Acute Adverse Events and Postoperative Complications in a Randomized Trial of Preoperative Short-course Radiotherapy Versus Long-course Chemoradiotherapy for T3 Adenocarcinoma of the Rectum: Trans-Tasman Radiation Oncology Group Trial (TROG 01.04). Ann. Surg. 2017, 265, 882-888. [CrossRef]

30. Bujko, K.; Nowacki, M.P.; Nasierowska-Guttmejer, A.; Michalski, W.; Bebenek, M.; Pudełko, M.; Kryj, M.; Olędzki, J.; Szmeja J.; Słuszniak, J.; et al. Sphincter preservation following preoperative radiotherapy for rectal cancer: Report of a randomised trial comparing short-term radiotherapy vs. conventionally fractionated radiochemotherapy. Radiother. Oncol. 2004, 72, 15-24. [CrossRef] [PubMed]

31. Bromham, N.; Kallioinen, M.; Hoskin, P.; Davies, R.J.; Guideline Committee. Colorectal cancer: Summary of NICE guidance. BMJ 2020, 368, m461. [CrossRef] [PubMed]

32. Allegra, C.J.; Yothers, G.; O'Connell, M.J.; Beart, R.W.; Wozniak, T.F.; Pitot, H.C.; Shields, A.F.; Landry, J.C.; Ryan, D.P.; Arora, A.; et al. Neoadjuvant 5-FU or Capecitabine Plus Radiation With or Without Oxaliplatin in Rectal Cancer Patients: A Phase III Randomized Clinical Trial. J. Natl. Cancer Inst. 2015, 107, djv248. [CrossRef] [PubMed]

33. Kwakman, J.J.M.; Simkens, L.H.J.; van Rooijen, J.M.; van de Wouw, A.J.; ten Tije, A.J.; Creemers, G.J.M.; Hendriks, M.P.; Los, M.; van Alphen, R.J.; Polée, M.B.; et al. Randomized phase III trial of S-1 versus capecitabine in the first-line treatment of metastatic colorectal cancer: SALTO study by the Dutch Colorectal Cancer Group. Ann. Oncol. 2017, 28, 1288-1293. [CrossRef]

34. Sadahiro, S.; Suzuki, T.; Tanaka, A.; Okada, K.; Kamijo, A.; Murayama, C.; Akiba, T.; Nakayama, Y. Phase I/II study of preoperative concurrent chemoradiotherapy with S-1 for locally advanced, resectable rectal adenocarcinoma. Oncology 2012, 81, 306-311. [CrossRef]

35. Sacdalan, D.B.; Lucero, J.A.; Sacdalan, D.L. Prognostic utility of baseline neutrophil-to-lymphocyte ratio in patients receiving immune checkpoint inhibitors: A review and meta-analysis. Onco Targ. Ther. 2018, 11, 955-965. [CrossRef] [PubMed]

36. Gavriilidis, P.; Wheeler, J.; Spinelli, A.; de'Angelis, N.; Simopoulos, C.; Saverio, S.D. Robotic vs laparoscopic total mesorectal excision for rectal cancers: Has a paradigm change occurred? A systematic review by updated meta-analysis. Colorectal Dis. 2020, 22, 1506-1517. [CrossRef] [PubMed]

37. Oikonomou, C.; Gourgiotis, S.; Cirocchi, R.; Piagkou, M.; Protogerou, V.; Troupis, T.; Biondi, A.; Sileri, P.; Filippou, D.; PelvicNeuroanatomy Collaborating Group; et al. Re-exploring the pelvic neuroanatomy from a new perspective and a potential guidance for TaTME: A “bottom-up” approach. Updates Surg. 2021, 73, 503-512. [CrossRef] [PubMed] 